PROCEEDINGS OF THE

AMERICAN MATHEMATICAL SOCIETY

Volume 127, Number 8, Pages 2245-2248

S 0002-9939(99)04820-0

Article electronically published on April 9, 1999

\title{
NAKAI'S CONJECTURE FOR VARIETIES SMOOTHED BY NORMALIZATION
}

\author{
WILLIAM N. TRAVES
}

(Communicated by Wolmer V. Vasconcelos)

\begin{abstract}
The notion of D-simplicity is used to give a short proof that varieties whose normalization is smooth satisfy Ishibashi's extension of Nakai's conjecture to arbitrary characteristic. This gives a new proof of Nakai's conjecture for curves and Stanley-Reisner rings.
\end{abstract}

\section{INTRODUCTION}

Nakai's conjecture concerns a very natural question: can differential operators detect singularities on algebraic varieties? On a smooth complex variety, it is well known that the ring of differential operators is generated by derivations. Nakai asked whether the converse holds: if the ring of differential operators is generated by derivations, is the variety smooth? In this paper, the notion of D-simplicity is used to give a short proof that varieties whose normalization is smooth satisfy Ishibashi's extension [2] of Nakai's conjecture to arbitrary characteristic. For example, any variety whose irreducible components are smooth satisfies Nakai's conjecture, giving a proof that Nakai's conjecture holds for Stanley-Reisner rings. Furthermore, this gives a simple new characteristic-independent proof of Nakai's conjecture for curves. The argument is quite short and rederives characteristic-dependent results of Mount and Villamayor (characteristic zero) [4] and Ishibashi (prime characteristic) [2].

\section{DEFINITIONS AND NOTATION}

Let $X=\operatorname{Spec}(R)$ be an affine algebraic variety defined over a field $k$ of characteristic zero. When $R$ is regular (that is, when $X$ is smooth), the ring of differential operators $D(R / k)$ (see $E G A$ [1] or [3]) equals $\operatorname{der}(R / k)$, the $R$-subalgebra generated by the derivations (see McConnell and Robson [3, Corollary 15.5.6]). Nakai conjectured that this condition characterizes nonsingularity: $R$ is regular if and only if $\operatorname{der}(R / k)=D(R / k)$. Ishibashi [2] extended Nakai's conjecture to varieties defined over an arbitrary perfect field.

Received by the editors August 11, 1997 and, in revised form, November 10, 1997.

1991 Mathematics Subject Classification. Primary 13N10; Secondary 16S32.

Key words and phrases. Differential operators, Nakai's conjecture, D-simple.

I would like to thank Karen E. Smith for many helpful discussions. This research has been partially funded by NSERC, the NSF, the Massachusetts Intstitute of Technology and the University of Toronto. 
A Hasse-Schmidt derivation $\Delta=\left\{\delta_{n}\right\}_{n=0}^{\infty} \subseteq \operatorname{End}_{k}(R)$ is a collection of $k$-linear endomorphisms of $R$ such that $\delta_{0}=i d_{R}$ and

$$
\delta_{n}(a b)=\sum_{i+j=n} \delta_{i}(a) \delta_{j}(b) .
$$

For example, if $R$ has characteristic zero and $d$ is a derivation, $\delta_{n}=\frac{1}{n !} d^{n}$ determines a Hasse-Schmidt derivation. Let $H S(R / k)$ be the $R$-algebra generated by the components $\delta_{n}$ of Hasse-Schmidt derivations on $R$. HS $\left.R / k\right)$ is a subalgebra of $D(R / k)$ and if $R$ has characteristic zero, $\operatorname{der}(R / k)=H S(R / k)$. In characteristic zero Grothendieck $[1,16.11 .2$ and 17.12.4] showed that when $R$ is smooth over $k$, $H S(R / k)=D(R / k)$; a proof in arbitrary characteristic can be found in Traves [9]. Ishibashi's extension ${ }^{1}$ of Nakai's conjecture is that $R$ is smooth over $k$ if and only if $H S(R / k)=D(R / k)$. The main result of this paper is that varieties whose normalization is smooth satisfy this extension of Nakai's conjecture.

\section{PRELIMINARY RESULTS}

Let $R$ be a reduced algebra of finite type over a perfect field $k$. For a reduced ring $R$, the normalization $R^{\prime}$ of $R$ is the integral closure of $R$ in its total ring of quotients, $L=S^{-1} R$, where $S$ is the multiplicative set of nonzerodivisors in $R$. The conductor of $R^{\prime}$ into $R$ is

$$
C=\left\{c \in R: c R^{\prime} \subseteq R\right\} .
$$

The conductor is an ideal of both $R$ and $R^{\prime}$.

Lemma 1. (1) The conductor is $H S(R / k)$-stable.

(2) Powers of $H S(R / k)$-stable ideals are $H S(R / k)$-stable.

Proof. Part (1) is well-known: see Seidenberg [6, Corollary on page 169 and section 5]. To establish (2) it suffices to show that for $a_{1}, \ldots, a_{s}$ in the $H S(R / k)$-stable ideal $I$ and $\Delta=\left\{\delta_{n}\right\}$ a Hasse-Schmidt derivation, $\delta_{n}\left(a_{1} \cdots a_{s}\right) \in I^{s}$. Now

$$
\delta_{n}\left(a_{1} \cdots a_{s}\right)=\sum_{i+j=n} \delta_{i}\left(a_{1}\right) \delta_{j}\left(a_{2} \cdots a_{s}\right)
$$

and the claim follows by induction on $s$.

Lemma 2. The conductor is not contained in any minimal prime of $R$.

Proof. The conductor $C$ equals $A n n_{R}\left(\frac{R^{\prime}}{R}\right)$. If $C \subseteq P$ with $P$ a minimal prime of $R$, then, since $\operatorname{Supp}\left(\frac{R^{\prime}}{R}\right)=\mathbb{V}\left(\operatorname{Ann}\left(\frac{R^{\prime}}{R}\right)\right), P \in \operatorname{Supp}\left(\frac{R^{\prime}}{R}\right)$. Localizing the exact sequence of $R$-modules

$$
0 \rightarrow R \rightarrow R^{\prime} \rightarrow \frac{R^{\prime}}{R} \rightarrow 0
$$

at the minimal prime $P$ gives an exact sequence

$$
0 \rightarrow R_{P} \rightarrow R_{P}^{\prime} \rightarrow\left(\frac{R^{\prime}}{R}\right)_{P} \rightarrow 0
$$

But since $P$ is a minimal prime of a reduced ring, $R_{P}$ is a field and the normalization map $R_{P} \rightarrow R_{P}^{\prime}$ is an isomorphism. This forces $\left(\frac{R^{\prime}}{R}\right)_{P}=0$, a contradiction. So $C$ is not contained in any minimal prime of $R$.

\footnotetext{
${ }^{1}$ Actually, Ishibashi requires that $k$ be algebraically closed and conjectures $R$ regular, but it is clear that smoothness is the relevant notion
} 
Lemma 3. If $R$ is a domain that is smooth over $k$, then $R$ is $D(R / k)$-simple.

Proof. Since $k$ is perfect, $R$ is regular. In the characteristic zero case, it is well known that $R$ is $D(R / k)$ )-simple (see McConnell and Robson [3, Theorem 15.3.8 and Corollary 15.5.6]). For the prime characteristic case, note that since $R$ is regular, $R$ is strongly F-regular and since $R$ is an algebra of finite type over a perfect field, $R$ is F-finite. Smith [7, Theorem 2.2] has shown that strongly F-regular F-finite domains are $D(R / k)$-simple.

\section{NAKAI'S CONJECTURE}

Theorem 4. Let $R$ be a reduced $k$-algebra of finite type and let $R^{\prime}$ be its integral closure in its total ring of quotients. If $R^{\prime}$ is a product of D-simple rings and $H S(R / k)=D(R / k)$, then $R$ is normal.

Proof. Note that $R^{\prime}$ is isomorphic to $R_{1} \times \cdots \times R_{t}$, where $R_{i}$ is the normalization of $\frac{R}{P_{i}}$ with the $\left\{P_{i}\right\}$ ranging over the minimal primes of $R$. By Lemma 1 (1), $C$ is $H S(R / k)$-stable and by Lemma $1(2), C^{2}$ is also $H S(R / k)$-stable. Thus, $C^{2}$ is $D(R / k)$-stable.

Assume that $C^{2} \neq C$. Since $C$ is not contained in any of the minimal primes $P_{i}$ of $R$, there are elements $c_{i} \in C \backslash P_{i}$ with some $c_{j} \notin C^{2}$. To see this, take $x \in C \backslash C^{2}$ and note that $x \neq 0 \Rightarrow x \notin \bigcap P_{i} \Rightarrow x$ is not in some $P_{j}$. Set $c_{j}=x$ and pick the other $c_{i} \in C \backslash P_{i}$. Let $c=\left(c_{1}, \ldots, c_{t}\right) \in R^{\prime}$, after identifying $R^{\prime}$ with the product in the first paragraph. Then $c \in C \backslash C^{2}$ and $c$ is nonzero in each component. By the D-simplicity of each of the $R_{i}$, there is an operator $\theta=\left(\theta_{1}, \ldots, \theta_{t}\right) \in D\left(R_{1}\right) \times \cdots \times D\left(R_{t}\right)$, such that $\theta\left(c^{2}\right)=1$. If each $\theta_{i} \in D\left(R_{i}\right)$ is an operator of order $\leq n_{i}$ then $\theta=\left(\theta_{1}, \ldots, \theta_{t}\right)$ maps $R^{\prime}$ to itself and $\theta$ is a differential operator of order $\leq n=\max \left(n_{i}\right)$. Thus, $\theta \in D\left(R^{\prime}\right)$ and $c \theta \in D(R)$. Now $(c \theta)\left(c^{2}\right)=c \notin C^{2}$, contradicting the fact that $C^{2}$ is $D(R / k)$-stable. This forces $C^{2}=C$.

In fact, $C=R$. Indeed, if $C$ is contained in a maximal ideal $m$, then $C_{m}=$ $C_{m}^{2} \subset m C_{m} \subset C_{m}$, so $m C_{m}=C_{m}$. Now Nakayama's lemma forces $C_{m}=0$. But then $C$ must consist of zero divisors, contradicting Lemma 2. So $C=R$ and $R$ is normal.

Theorem 5. If $H S(R / k)=D(R / k)$ and the normalization $R^{\prime}$ of $R$ is smooth over $k$, then $R$ is smooth over $k$.

Proof. Since the normalization $R^{\prime}$ of $R$ is a product of smooth domains, $R^{\prime}$ is a product of $D$-simple rings (by Lemma 3 ). The result now follows from Theorem 4.

This theorem says that the ring of differential operators of a singular complex variety whose normalization is smooth is not generated by derivations. Thus, $D(R / \mathbb{C})$ is complicated even for very mild singularities (those that can be resolved by normalization). We expect that $D(R / \mathbb{C})$ will become more complicated as the singularities become worse. This provides further evidence for Nakai's conjecture.

The theorem shows that Nakai's conjecture holds for reduced varieties smoothed by normalization; for example, curves.

Corollary 6. Let $R$ be a reduced $k$-algebra of finite type, where $k$ is a perfect field. If $R$ is 1-dimensional and $H S(R / k)=D(R / k)$, then $R$ is smooth over $k$. In 
particular, if $R$ is a domain of characteristic 0 and $\operatorname{der}(R / k)=D(R / k)$, then $R$ is regular.

Theorem 5 can also be used to recover a result due to Schreiner [5] (also, see Traves [8]): Nakai's conjecture holds for Stanley-Reisner rings (that is, for subvarieties of $\mathbb{A}_{k}^{N}$ which are the union of coordinate subspaces). More generally, the theorem implies that Nakai's conjecture holds for varieties all of whose components are smooth, as remarked by Lazarsfeld.

Corollary 7. Let $R$ be a reduced $k$-algebra of finite type, where $k$ is a perfect field. If $H S(R / k)=D(R / k)$ and if $\frac{R}{P}$ is smooth over $k$ for each minimal prime $P$ of $R$, then $R$ is smooth over $k$. In particular, if $R$ is a Stanley-Reisner ring and $H S(R / k)=D(R / k)$, then $R$ is a polynomial ring.

Both Corollary 6 and Corollary 7 follow immediately from Theorem 5: just observe that the normalization of $X=\operatorname{Spec}(R)$ is isomorphic to the disjoint union of the normalization of the components of $X$, each of which is smooth over $k$.

\section{REFERENCES}

[1] Dieudonné, J. and Grothendieck, A. Élements de géométrie algèbrique IV. Publ. Math. I.H.E.S. 32 (1967). MR 39:220

[2] Ishibashi, Yasunori. An Analogue of Nakai's Conjecture. Communications in Algebra 13 (1985), no. 3, 575-584. MR 86e: 13005

[3] McConnell J. C., and Robson, J. C. Noncommutative Noetherian Rings. Wiley, New York, 1987. MR 89j: 16023

[4] Mount, Ken R. and O. E. Villamayor. On a Conjecture of Y. Nakai. Osaka J. Math. 10 (1973), 325-327. MR 48:6073

[5] Schreiner, A. On a Conjecture of Nakai. Arch. Math. 62 (1994), 506-512. MR 95f:13033

[6] Seidenberg, A. Derivations and Integral Closure. Pacific J. Math. 16 (1966), no. 1, 167-173. MR 32:5686

[7] Smith, K. E. The D-module Structure of F-split Rings. Mathematical Research Letters. 2 (1995), 377-386. MR 96j:13024

[8] Traves, William N. Differential Operators on Monomial Rings. Journal of Pure and Applied Algebra. To appear.

[9] Traves, William N. Differential Operators and Nakai's Conjecture. Ph.D. Thesis. University of Toronto (1998).

Department of Mathematics, University of Toronto, 100 St. George Street, 4th Floor, Toronto, Ontario, Canada M5S 3G3

E-mail address: traves@math.toronto.edu 